Original Paper http://ajol.info/index.php/ijbcs http://indexmedicus.afro.who.int

\title{
Analyse des besoins en mécanisation agricole basée sur les logiques paysannes dans les pôles de développement agricole du Bénin
}

\author{
Cossi Léonard HINNOU ${ }^{*}$, Vidjannangni Dieudonné AGBOTRIDJA ${ }^{2}$ et \\ René Nestor AHOYO ADJOVI ${ }^{3}$ \\ ${ }^{I}$ Centre de Recherches Agricoles Centre (CRAC), Institut National des Recherches Agricoles du Bénin \\ (INRAB), 01 BP 884 Cotonou, Bénin. \\ ${ }^{2}$ Groupe d'Experise en Recherche et Développment Intégré (GERDI), 02 BP 833 Porto-Novo, Cotonou, \\ Bénin. \\ ${ }^{3}$ Institut National des Recherches Agricoles du Bénin (INRAB), 01 BP 884 Cotonou, Bénin. \\ *Corresponding author, E-mail: hicoll77@yahoo.fr, Tel: (+229) 97738904
}

\begin{abstract}
Received: $14-11-2020$
Accepted: 08-04-2021

Published: 30-04-2021

\section{RESUME}

L'agriculture béninoise est tributaire depuis plusieurs années de l'utilisation des outils rudimentaires malgré les efforts consentis par les gouvernants successifs. L'étude a été basée sur une approche participative aux fins d'identifier de concert avec les producteurs et transformateurs, les besoins en mécanisation agricole pré et post-récolte. Elle a été conduite à l'aide d'un guide d'entretien semi-structuré auprès de 129 producteurs et 66 transformateurs au niveau de 13 villages au Bénin. Les résultats ont montré que malgré leur satisfaction, les utilisateurs des matériels et équipements agricoles ont exprimé des besoins pour un renforcement de la mécanisation agricole. Ainsi, les principales opérations pré-récolte à mécaniser étaient la préparation du sol, le labour, l'herbicidage, le désherbage/sarclage et la récolte. Pour les opérations post-récolte, les besoins en équipements concernaient le décorticage/égrenage, l'épluchage, le battage/despathage, le broyage, le pressage et le conditionnement/emballage. Par ailleurs, les prototypes des outils préférés par les producteurs/transformateurs devraient présenter une puissance élevée et être capables de réduire le taux de perte puis d'améliorer la qualité du produit. A cela, doivent s'ajouter le temps de travail par jour avoisinant $5 \mathrm{~h}$, une durée d'amortissement de huit ans et deux opérateurs au plus pour leur fonctionnement.
\end{abstract}

(C) 2021 International Formulae Group. All rights reserved.

Mots clés : Bénin, opérations pré et post récoltes, préférences, représentation sociale.

\section{Analysis of farming mechanization needs based on the logics of farmers in the Benin agricultural development poles}

\begin{abstract}
Since years, Benin agriculture has been relying on the utilization of rudimentary equipment despite the efforts made by successive governments. This study was based on a participatory approach to identify with producers and processors, the needs for agricultural mechanization pre- and post-harvest. For this purpose, the study was conducted using a semi-structured interview guide with 129 producers and 66 processors distributed
\end{abstract}


accross thirteen (13) villages of Benin. The results obtained show that despite their satisfaction, the users of agricultural machinery have expressed needs for an enhacement of agricultural mechanization. Thus, the main pre-harvest operations to be mechanized were soil preparation, plowing, herbicidage, weeding / weeding and harvesting. For post-harvest operations, equipment needs include shelling / ginning, peeling, threshing / shucking, grinding, pressing and packing. However, the agricultural equipment and materials desired by the producers and processors must have a high power and a capacity to reduce the rate of loss and improvement of the quality of the final product. Additionally, an average working time of about five (5) hours, an average replacement period of eight (8) years and two (2) operators at most for their functioning.

(C) 2021 International Formulae Group. All rights reserved.

Keywords: Benin, pre and post harvest operations, préférences, social representation.

\section{INTRODUCTION}

L'agriculture béninoise joue un rôle prépondérant dans l'amélioration du développement humain en raison de ses interactions multisectorielles (Hinnou, 2018). Aussi, selon FAO et CEDEAO (2018), le secteur agricole constitue la principale source de devises et de renforcement de l'économie béninoise. En effet, il contribue à plus de $70 \%$ des emplois et environ à $33 \%$ au Produit Intérieur Brut, et fournit $75 \%$ des recettes d'exportation d'origine intérieure (PNUD, 2015; INSAE, 2016; FAO et CEDEAO, 2018). Cependant, l'agriculture béninoise reste tributaire de l'utilisation des outils traditionnels. Ainsi, la force de travail dans le secteur agricole dépend dans une écrasante majorité de la force physique avec l'utilisation d'outils manuels comme la houe pour exécuter les différentes opérations culturales (Silou, 2003). Les outils manuels ainsi utilisés présentent souvent des limites tant sur le plan énergique que celui du rendement obtenu induisant une faible productivité des exploitations agricoles (Side, 2003; Ahouandjinou et al., 2010). Cette faible productivité résulte notamment de la faible mécanisation du secteur (Ficou, 2017). Pour rémédier à cette situation, le Bénin a œuvré à travers plusieurs programmes accompagnés par des structures sous la tutelle du Ministère de l'Agriculture de l'Elevage et de la Pêche (MAEP), des structures d'appui au conseil agricole, des Organisations Non Gouvernementales (ONG), des projets, etc. pour faire sortir son agriculture de l'ornière. Pour cela, plusieurs localités ont bénéficié d'appui des structures en matière d'équipements/matériels agricoles (Ahouandjinou et al., 2010 ; Atidegla et al., 2017). Malheureusement, les résultats de la plupart des interventions sont mitigiés et l'écart entre les effets escomptés et ceux obtenus paraît trop grand (Balse et al., 2015). Ainsi, les différents problèmes auxquels est confrontée la mécanisation de l'agriculture béninoise ont eu pour corollaire le mauvais entretien des équipements, la vétusté et le nonrenouvellement des équipements mis à la disposition des producteurs, la non disponibilité des pièces de rechange sur place, la complexité à l'utilisation, le coût élevé etc (Silou et al., 2003). Toutes ces raisons justifient le recours des producteurs aux outils adaptés à la spécificité de l'agriculture béninoise et aux caractéristiques préférées (Adekunle et al., 2015).

Pour pallier ces insuffisances et contraintes entravant la durabilité, la productivité de l'agriculture et de limiter les pertes pré et post-récolte, il est primordial de déterminer les besoins des acteurs en matériels et équipements pré et post-récolte en lien avec leurs perceptions. En effet, la plupart des études antérieures en lien avec la problématique se sont interessées spécifiquement aux besoins des producteurs sans intégrer leurs réprésentations de la mécanisation agricole comme celle de Agbangba et al. (2018). En conséquence, les innovations mises au point et diffusées dans le secteur agricole ont du mal à se faire une place dans le milieu paysan, bien qu'améliorées et jugées techniquement plus performantes 
(Hinnou, 2018). D'où la pertinence de la présente étude qui vise à analyser les besoins en matériels et équipements agricoles des producteurs et transformateurs en se basant sur les logiques socialement construites de la mécanisation agricole. In fine, la recherche a conduit à la détermination des prototypes des matériels et équipements préférés par les exploitants agricoles dans les Pôles de Développement Agricole du Bénin.

Pour analyser les besoins en mécanisation des producteurs et transformateurs agricoles, cette étude s'est basée sur la théorie du choix rationnel. La théorie du choix rationnel est axée sur trois postulats (Boudon, 2002). Le premier pose que tout phénomène social résulte de la combinaison d'actions, de croyances ou d'attitudes individuelles. Le second postulat tend à « comprendre » les actions, croyances et attitudes de l'acteur individuel. Quant au dernier postulat, il argue que l'acteur adhère à une croyance, ou entreprend une action parce qu'elle a du sens pour lui, en d'autres termes que la cause principale des actions, croyances, etc. du sujet réside dans le sens qu'il leur donne, plus précisément dans les raisons qu'il a de les adopter. Cette théorie aide alors à mieux comprendre que les besoins en matériels et équipements agricoles exprimés par les producteurs et tranformateurs résultent des expériences, croyances, actions et attitudes de ces derniers. Cependant, il leur est souvent difficile d'établir une liste exhaustive de tous les matériels et équipements dont ils ont besoin (Silou, 2003). A cet effet, il appartient aux technologues d'effectuer une évaluation des besoins en technologie agricole de manière fine et à l'aide de méthodologies éprouvées (Silou, 2003) car il en va de l'attractivité et du développement du secteur agricole. Aussi, faut-il noter qu'en s'appuyant sur les besoins exprimés par les producteurs en équipements, les technologues ont l'obligation de proposer à ces derniers des machines adaptées aux réalités locales. En effet, les versions bon marché et techniquement peu sophistiquées de tracteurs à deux ou quatre roues par exemple fournissent la force de traction nécessaire aux petits exploitants agricoles (Adekunle et al., 2015).
Les machines agricoles sont donc nécessaires pour mener à bien les activités pré et postrécolte afin d'éviter d'énormes pertes de production et de transformation. C'est dans ce cadre que s'inscrit, le cadre analytique (Figure $1)$.

\section{MATERIEL ET METHODES \\ Site de recherche et échantillonnage}

L'étude a été conduite au niveau de treize (13) sites de Recherche-Développement répartis sur l'ensemble des sept (7) Pôles de Développement Agricole (PDA) du Bénin. Le Pôle de Développement Agricole est une zone géographiquement et agro-écologiquement bien définie à vocation agricole spécifique. C'est un nouveau dispositif portant sur la territorialisation du développement agricole en ligne avec le schéma d'aménagement du territoire dans un but d'efficacité, d'obligation de performance, de promotion des filières, d'utilisation optimale des ressources publiques. C'est un «cadre de mise en œuvre opérationnelle des politiques, programmes et projets de développement agricole ». L'échantillonnage des villages d'enquête et des unités d'analyse a été basé sur une approche mixte (raisonnée combinée à l'aléatoire) qui avait tenu compte de l'existence des contraintes relatives à la mécanisation des opérations pré et post-récoltes. Au total, 129 producteurs/productrices et 66 transformateurs/transformatrices ont été enquêtés dans la zone d'étude. La répartition de l'échantillon par Pôle de Développement Agricole et par commune est présentée dans le Tableau 1.

\section{Données collectées, méthodes de collecte et d'analyse des données}

Les données collectées dans le cadre de cette recherche sont relatives aux caractéristiques socio-économiques et démographiques des producteurs et transformateurs agricoles, aux représentations Socialement construites autour de la mécanisation agricole, le type et la nature des équipements pré et post-récolte utilisés, le degré de satisfaction des enquêtés par rapport à ces équipements, les besoins réels des 
producteurs et transformateurs en termes de mécanisation pré et post récolte en lien avec les opérations à mécaniser, les préférences en termes de caractéristiques (temps (heure) de travail par jour, temps (année) moyen de renouvèlement, nombre d'opérateurs, capacité ou puissance, réduction du taux de perte et amélioration de la qualité du produit final). Ces données avaient été collectées au moyen des focus group et des entretiens individuels. L'observation non participante a également été utilisée pour apprécier l'état des machines agricoles possédés par les acteurs interviewés. Les données ainsi collectées, ont été soumises à l'analyse de contenu thématique en continu en ce qui concerne les données qualitatives (Paillé et Mucchielli, 2013). Tandis que les données quantitatives ont fait l'objet des statistiques descriptives (moyenne, fréquence relative et écart-type) appuyées des tests statistiques (test de Kendall, W, test de Khideux).

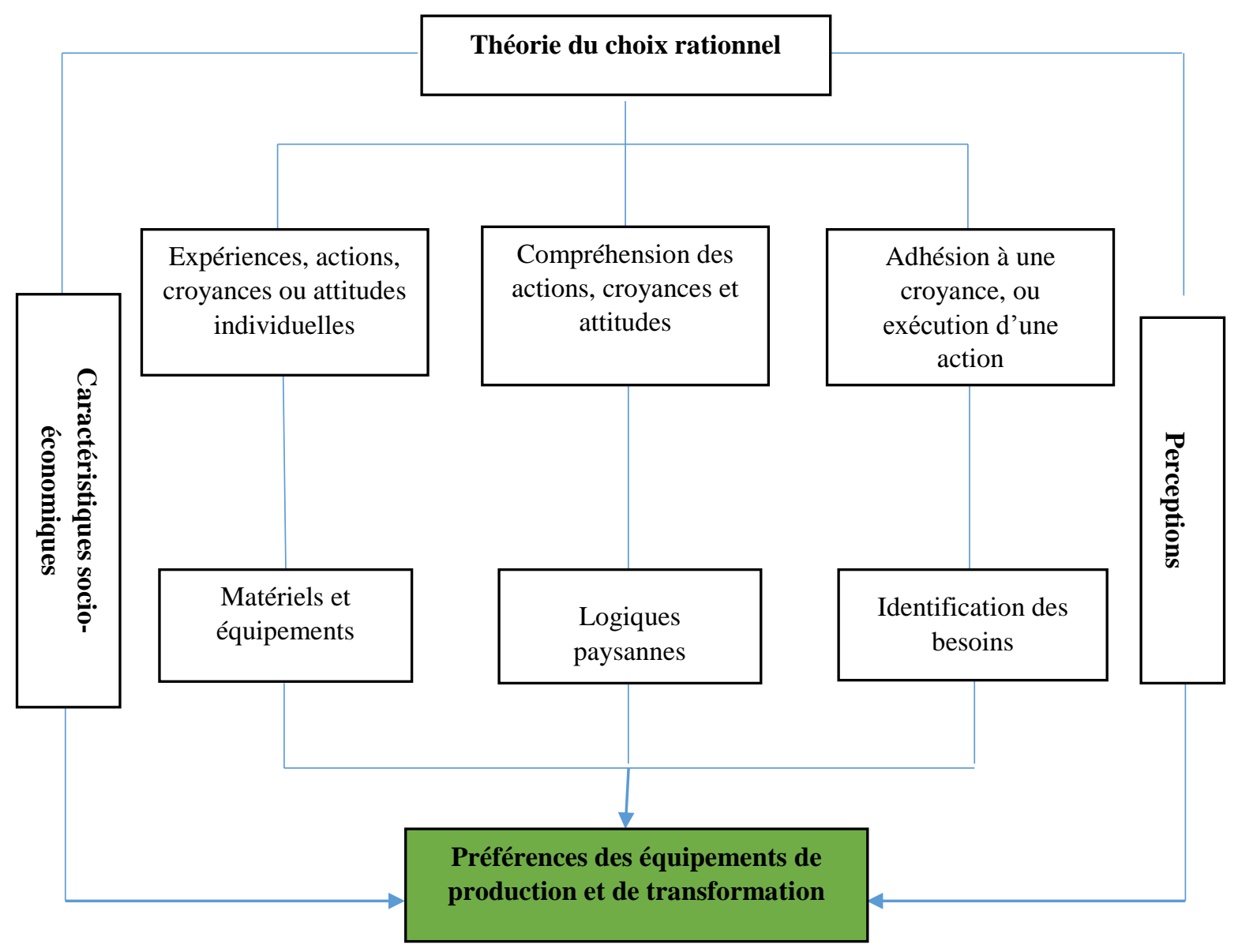

Figure 1 : Cadre analytique de la recherche. 
Tableau 1 : Répartition de l'échantillon par Pôle de Développement Agricole (PDA).

\begin{tabular}{|c|c|c|c|c|}
\hline \multirow{2}{*}{ PDA } & \multirow{2}{*}{ Communes } & \multirow{2}{*}{ Villages/sites } & \multicolumn{2}{|c|}{ Effectif } \\
\hline & & & Producteur & Transformateur \\
\hline Pôle 1 : Vallée du Niger & Karimama, Gogounou & Birni-Lafia, Badou & 20 & 7 \\
\hline $\begin{array}{l}\text { Pôle 2 : Alibori Sud et } \\
\text { Borgou Nord }\end{array}$ & Péhunco, Boukombe & Soadou, Kouya & 20 & 13 \\
\hline $\begin{array}{l}\text { Pôle } 3 \text { : Atacora Ouest, } \\
\text { Borgou Sud et Donga }\end{array}$ & Ouaké & Awanla-Kpéloudè & 11 & 5 \\
\hline Pôle 4 : Collines & $\begin{array}{l}\text { Dassa-Zoumé, Bantè, } \\
\text { Ouèssè }\end{array}$ & $\begin{array}{l}\text { Miniffi, Gbanlin } \\
\text { Akatakou }\end{array}$ & 30 & 21 \\
\hline Pôle 5 : Zou et Couffo & Aplahoué, Djakotomey & $\begin{array}{l}\text { Eglimey, } \\
\text { Zouzouvou }\end{array}$ & 18 & 6 \\
\hline Pôle 6 : Plateau & Kétou, Sakété & $\begin{array}{l}\text { Adakplamè, } \\
\text { Saharo-Nagot }\end{array}$ & 10 & 10 \\
\hline $\begin{array}{l}\text { Pôle } 7 \text { : Ouémé, } \\
\text { Atlantique et Mono }\end{array}$ & Tori-Bossito & Hayakpa & 20 & 4 \\
\hline Ensemble & & & 129 & 66 \\
\hline
\end{tabular}

\section{RESULTATS \\ Utilisation et satisfaction des producteurs et transformateurs par rapport aux matériels et équipements agricoles}

Les taux d'utilisation des équipements de production et de transformation sont relativement faibles (Figures 2 et 3). En effet, les principales opérations mécanisées sont le labour (53\% des producteurs), le désherbage (74\% des cas), la lutte contre les insectes/maladies (61\% des enquêtés), le décorticage/égrenage ( $36 \%$ des producteurs) et de transport (42\% des enquêtés). Par contre, la préparation du sol, le semis, la lutte contre les ravageurs, la récolte, le séchage et le conditionnement sont encore à l'étape manuelle (Figure 2). La tendance est presque la même en ce qui concerne les opérations de transformation. Seules les opérations de broyage et de pressage sont moyennement mécanisées par respectivement $64 \%$ et $34 \%$ des transformateurs enquêtés (Figure 3). Par contre, les opérations de râpage, de décorticage, de séchage et de conditionnement/emballage sont faiblement mécanisées. Cette situation s'explique par la non-disponibilité de ces matériels et équipements agricoles dans la zone d'étude et donc leur méconnaissance par les producteurs et transformateurs enquêtés.

Par ailleurs, le niveau de satisfaction des producteurs et des transformateurs par rapport aux équipements agricoles utilisés est présenté sur les Figures 4 et 5 . Il en ressort que la plupart des producteurs ayant utilisé des machines agricoles pour exécuter une opération culturale sont très satisfaits. Cependant, leur satisfaction de la mécanisation des opérations de préparation du sol (11\% des enquêtés), de labour ( $21 \%$ des cas) et de la lutte contre les ravageurs (22\% des producteurs) est peu mitigée. De même, bien que la satisfaction des transformateurs enquêtés soit globalement bonne, des efforts d'amélioration restent encore à faire au niveau des opérations de pressage, de râpage, de broyage, de décorticage et de conditionnement. Cette insatisfaction est due notamment aux caractéristiques (capacité, temps de travail, durée de renouvellement etc.) des matériels et équipements utilisés par ces derniers. Aussi, la qualité en termes de texture $\mathrm{du}$ produit obtenu reste-t-elle, dans certains cas, loin des attentes des enquêtés. 


\section{Constructions sociales des acteurs autour de la mécanisation agricole au Bénin}

La mécanisation agricole, perçue comme un objet ou un phénomène social, est sujette à des représentations socialement construites par les producteurs et les transformateurs des produits agricoles. Ainsi, en se basant sur la théorie existante, les construits sociaux des acteurs ont été regroupés en trois catégories: le niveau individuel, le niveau interpersonnel et le niveau idéologique. Le test de Kendall est significatif au seuil de $1 \%$ indiquant une concordance dans la hiérarchisation des représentations sociales faite par les producteurs et les transformateurs interviewés (Tableau 2).

$\mathrm{Au}$ niveau individuel, autant les producteurs que les transformateurs ont souligné que, sans la mécanisation, il est impossible d'améliorer le rendement agricole et même d'accroitre la superficie emblavée ou la quantité des produits tranformée. Cependant, les machines agricoles sont appréhendées comme des moyens de luxe/réservés aux riches du fait que son accès est subordonné à la disponibilité financière soit pour l'achat ou pour la location. «Le labour avec le tracteur nous revient très cher, il faut savoir que labourer un ha de terre coûte 30000 FCFA (environ 47 euros) ", avait déclaré un producteur.

$\mathrm{Au}$ niveau interpersonnel, le renforcement des relations sociales, le privilège dans le groupe social/ reseau social et la participation aux prises de décision de production constituent les trois principales representations des producteurs et des transformateurs de la mécanisation agricole. En effet, selon les enquêtés, lorsqu'un producteur dispose d'une machine agricole, il accroît son potentiel social et un grand nombre de producteurs/transformateurs le sollicite pour des prestations de service rémunérées ou sous forme de prêt. Aussi, la personne détentrice de la machine agricole dispose-t-elle d'un privilège au sein de son groupe social du point de vue de pouvoir décisionnel. Par contre, la solidarité est une vertu partagée par la plupart des enquêtés du milieu d'étude. Autrement, la mécanisation ne saurait donc être un facteur d'exclusion sociale.

$\mathrm{Au}$ niveau idéologique, la grande emblavure, le gain de temps/rapidité du travail et la diminution des blessures sont les évocations majeures autour de la mécanisation agricole. En effet, les producteurs et transformateurs sont plus exposés aux accidents de travail avec l'utilisation des équipements traditionnels. "Quand tu travailles avec la houe, tu peux te blesser facilement car elle peut taper une pierre et revenir te heurter la jambe. De plus, tu ne sais pas ce qu'il y a sous le sol ou dans les herbes. Un serpent peut s'y cacher et te mordre », avait affirmé un enquêté. De même, l'utilisation des machines agricoles permet non seulement de gagner du temps tout en améliorant sa capacité de production tant qualitativement que quantitativement. Cependant, certains producteurs interviewés avaient affirmé que l'utilisation des machines agricoles contribue à appauvrir le sol. Cette construction sociale de la mécanisation trouve son fondement dans la profondeur du labour qui appauvrit, à long terme, le sol : "Le tracteur remue trop le sol, ce qui laisse assez de traces et appauvrit le sol. La production sur ces sols labourés avec le tracteur décroît progressivement». A contrario, une frange des producteurs estiment que la mécanisation ne saurait être un moyen d'appauvrissement du sol puisqu'elle ne facilite que la réalisation des opérations.

\section{Préférences des acteurs en matériels/équipements agricoles : un essai de construction des prototypes :}

Les principales opérations pré-récolte à mécaniser étaient la préparation du sol, le labour, le semis/repiquage, l'herbicidage, le désherbage/sarclage et la récolte (Figures 6). En effet, ces opérations nécessitent d'énormes efforts pour leur réalisation. Ainsi, plus de la 
moitié des producteurs enquêtés avaient exprimé le besoin de mécanisation de la préparation du sol (64\% des enquêtés), du labour (88\%), du semis (61\%), du désherbage (64\%), de l'application de l'herbicide (67\%), de la lutte contre les insectes (58\%) et de la récolte (64\%) (Figure 6). Quant aux opérations post-récolte (tous produits confondus) à mécaniser figurent l'épluchage (39\%), le nettoyage/vannage (38\%), le lavage $(38 \%)$, le pressage $(58 \%)$, le broyage $(73 \%)$, le décorticage (39\%) et le conditionnement/emballage (44\%) (Figure 7). Evidemment, la plupart de ces opérations constituent souvent de véritables goulots d'étranglement pour l'atteinte des objectifs de production et de transformation.

En se référant au Tableau 3 relatif aux différentes préférences des outils, la majorité des producteurs et transformateurs avaient exprimé le besoin de disposer de materiels et équipements avec une puissance élevée. Toutefois, une analyse différenciée montre qu'au niveau de la production, seules les opérations de semis et d'application d'engrais nécessitent des équipements avec une puissance relativement faible. Les autres opérations pré-récolte doivent être exécutées avec des équipements de forte puissance du fait de l'intensité de travail et des efforts énormes que requièrent ces opérations. Au niveau de la transformation, certaines opérations (râpage, pressage, broyage, fermentation, torréfaction) exigent une certaine technicité et flexibilité dont une forte puissance ne saurait bien contrôler. Aussi, les producteurs et transformateurs enquêtés avaient-ils une préférence pour les matériels et équipements visant l'amélioration de la qualité du produit fini. Cette préférence était moins pour certaines opérations (le labour, l'application d'engrais et la lutte contre les ravageurs) dont l'infuence sur la qualité du produit fini était très peu perceptible. Par ailleurs, les producteurs préfèraient des matériels et équipements capables de fonctionner en moyenne pendant cinq heures par jour contre quatre heures pour les transformateurs. La durée de renouvellement avait été aussi un facteur important qui avait sous-tendu les préférences des producteurs et transformateurs agricoles dans la zone d'étude. En effet, plus un équipement a une durée de vie élevée, plus longtemps il servira. Ainsi, les enquêtés préfèraient en moyenne une durée de renouvèlement des équipements avoisinant huit années calendaires. Toutefois, étant confrontés régulièrement à des problemes de ravageurs, les producteurs avaient préfèré des équipements ayant une durée de vie moyenne de 11 ans. Dans la même logique, les équipements d'épluchage, de râpage, de pressage, de broyage et de décorticage/égrenage devraient avoir en moyenne une durée de renouvèlement pouvant atteindre neuf ans. Pour ce qui est du nombre d'opérateurs, les enquêtés avaient affiché une préférence pour des équipements nécessitant au plus deux opérateurs pour leur fonctionnement (manipulation). En effet, les équipements habituellement utilisés par les producteurs pour certaines opérations n'étaient pas assez grands pour nécessiter l'intervention de plus de deux opérateurs. Il en était de même pour l'opération de torréfaction, au niveau de la transformation, qui n'exigeait qu'un seul opérateur.

Globalement, trois prototypes d'équipements de production et de transformation en lien avec les perceptions des acteurs étaient à distinguer. Le premier prototype faisait référence à des matériels et équipements performants et éfficients. Le second prototype était celui des équipements et matériels de capacité moyenne. Quant au troisième prototype, il faisait état des matériels et équipements de faible capacité. 
C. L. HINNOU et al. / Int. J. Biol. Chem. Sci. 15(2): 536-549, 2021

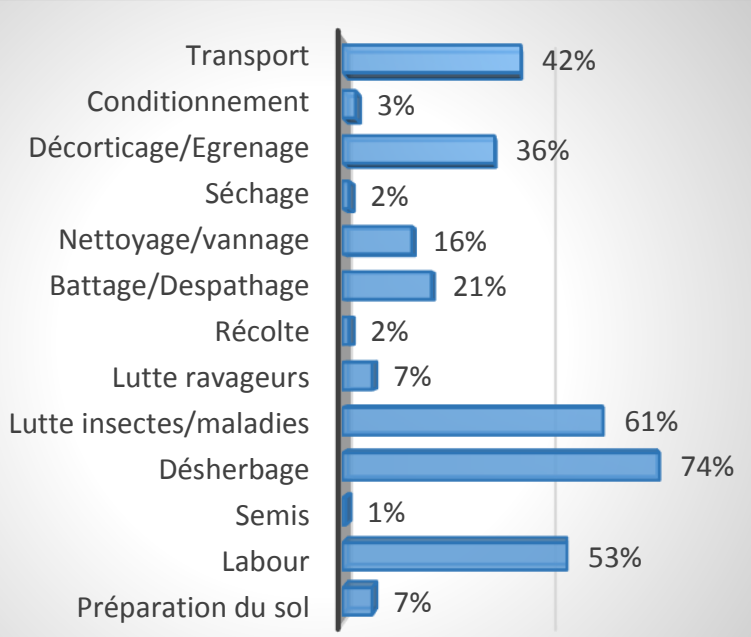

Figure 2 : Taux de mécanisation des opérations de production.

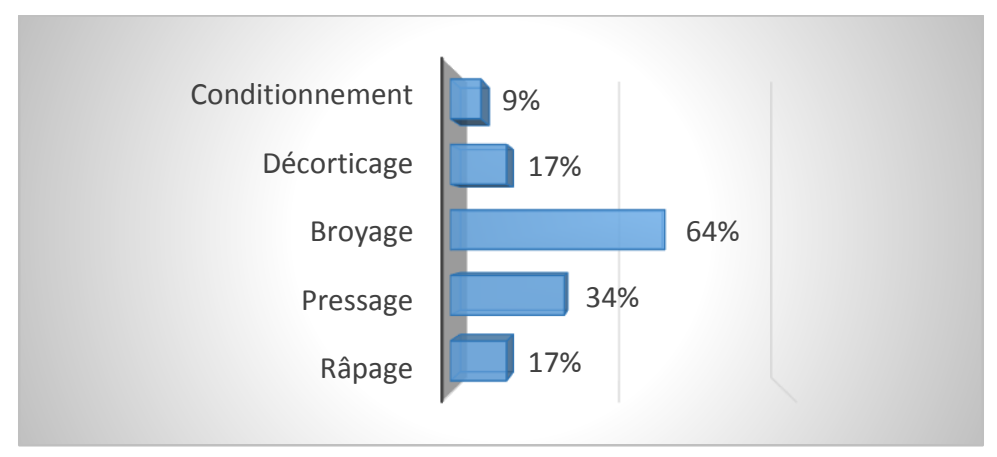

Figure 3: Taux de mécanisation des opérations de transformation.

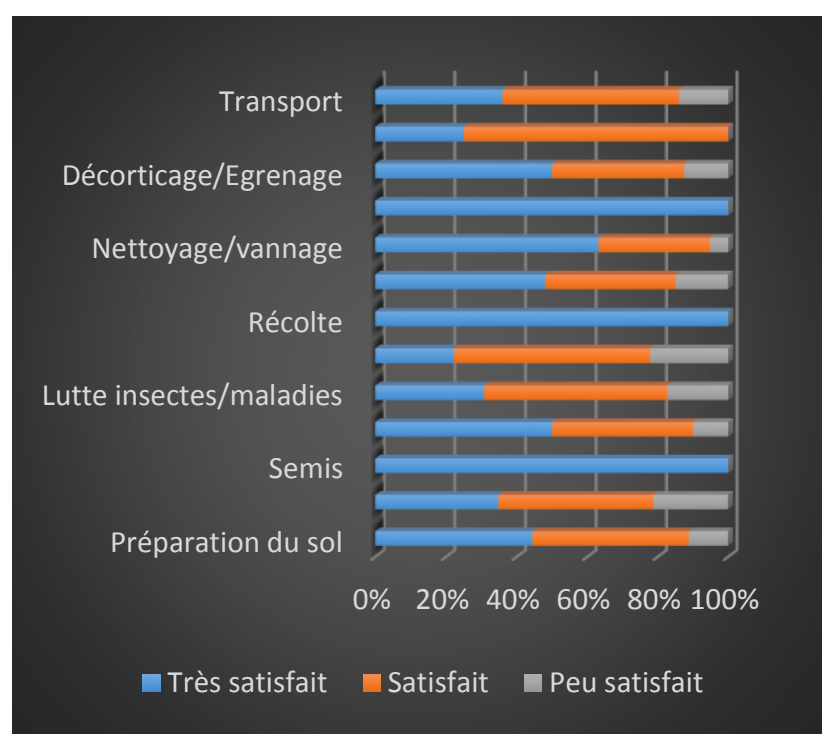

Figure 3 : Satisfaction des producteurs. 


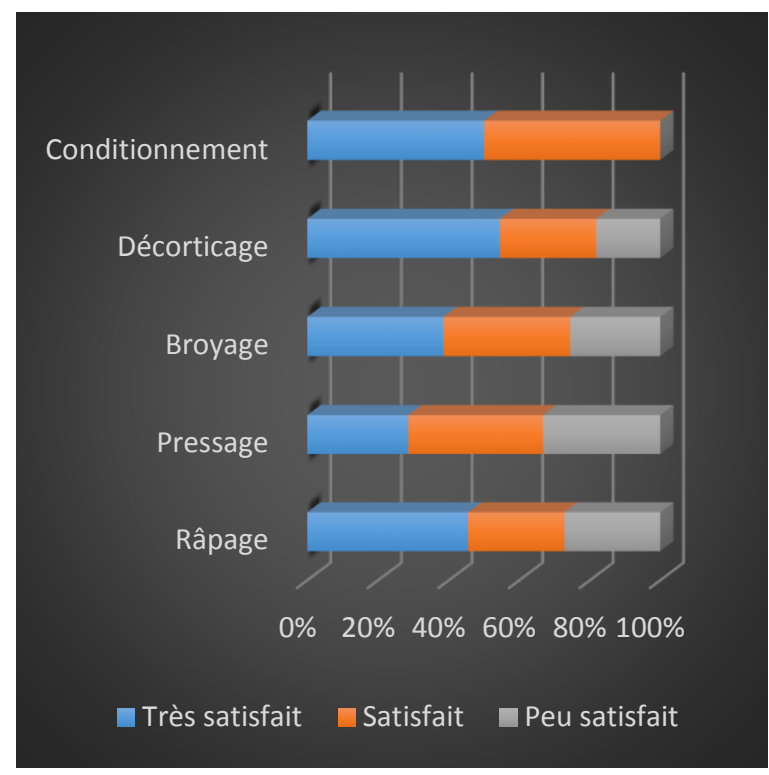

Figure 4 : Satisfaction des transformateurs.

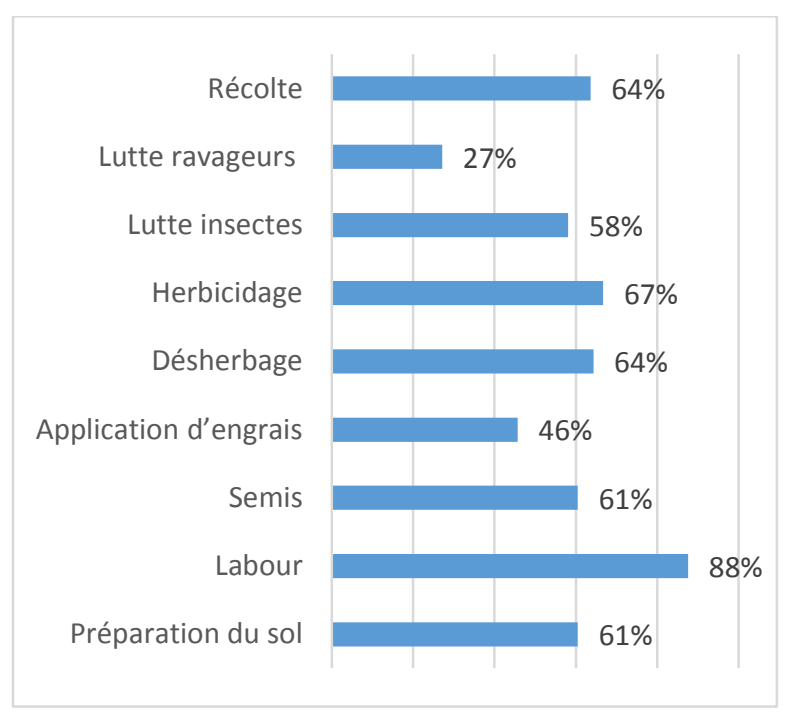

Figure 5: Opérations pré-récolte à mécaniser. 
Tableau 2 : Représentations sociales de la mécanisation agricole.

\begin{tabular}{|c|c|c|c|c|c|}
\hline \multirow[b]{2}{*}{ Niveau } & \multirow[b]{2}{*}{ Variables } & \multicolumn{2}{|c|}{ Producteurs } & \multicolumn{2}{|c|}{ Transformateurs } \\
\hline & & $\begin{array}{c}\text { Rang } \\
\text { moyen }\end{array}$ & Rang & $\begin{array}{c}\text { Rang } \\
\text { moyen }\end{array}$ & Rang \\
\hline \multirow{8}{*}{ Individuel } & Impossibilité d'amélioration du rendement agricole & 3,36 & 1 & 3,02 & 1 \\
\hline & Impossibilité d'accroître la superficie emblavée & 3,36 & 2 & 3,51 & 2 \\
\hline & Moyen de luxe/réservé aux « riches » & 3,37 & 3 & 4,08 & 4 \\
\hline & Dépendance totale de la main d'œuvre, des autres & 4,04 & 4 & 4,21 & 5 \\
\hline & La chose/pratique des blancs « yo vo nu » & 4,15 & 5 & 4,92 & 7 \\
\hline & Sans la mécanisation, pas de production & 4,39 & 6 & 3,61 & 3 \\
\hline & Abandon des outils traditionnels & 5,34 & 7 & 4,66 & 6 \\
\hline & Kendall W & $0,114 * * *$ & & $0,098 * * *$ & \\
\hline \multirow{6}{*}{ Interpersonnel } & Renforce les relations sociales & 1,57 & 1 & 1,51 & 1 \\
\hline & Privilège dans le groupe / réseau social & 2,51 & 2 & 2,58 & 2 \\
\hline & Participation aux prises de décision de production & 2,97 & 3 & 3,17 & 3 \\
\hline & Objet de fragilisation des liens sociaux & 3,78 & 4 & 3,8 & 4 \\
\hline & Facteur d'exercice social & 4,18 & 5 & 3,95 & 5 \\
\hline & Kendall W & $0,428 * * *$ & & $0,408 * * *$ & \\
\hline \multirow{8}{*}{ Idéologie } & Grande emblavure & 1,98 & 1 & NA & NA \\
\hline & Gain de temps/Rapidité du travail & 2,38 & 2 & 1,88 & 1 \\
\hline & Diminution des blessures & 3,45 & 3 & 3,24 & 4 \\
\hline & Amélioration du rendement & 3,52 & 4 & 2,86 & 3 \\
\hline & Diversification des cultures & 4,16 & 5 & NA & NA \\
\hline & Appauvrissement du sol & 5,5 & 6 & NA & NA \\
\hline & Grande transformation & NA & NA & 2,02 & 2 \\
\hline & Kendall W & $\mathbf{0 , 4 5 9} * * *$ & & $0,27 * * *$ & \\
\hline
\end{tabular}

*** signification au seuil de $1 \%(\mathrm{p}<0,01),{ }^{* *}(\mathrm{p}<0,05)$ et $*(\mathrm{p}<0,1)$.

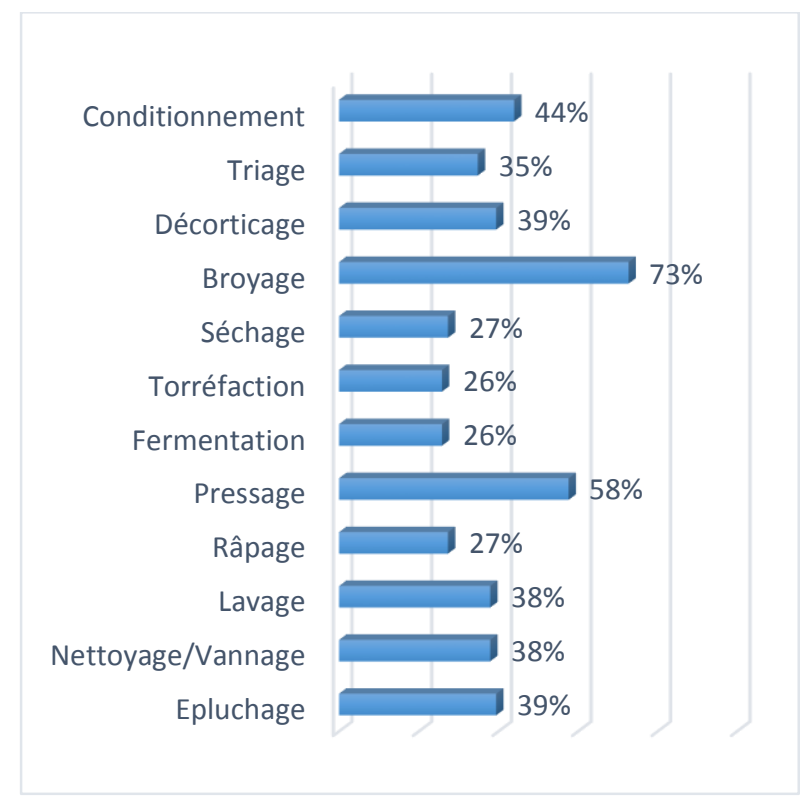

Figure 6: Opérations post-récolte à mécaniser. 
Tableau 3 : Prototypes des équipements de production et de transformation.

\begin{tabular}{|c|c|c|c|c|c|c|}
\hline Opérations & Puissance & $\begin{array}{c}\text { Réduction } \\
\text { du taux } \\
\text { de perte } \\
\end{array}$ & $\begin{array}{c}\text { Amélioration } \\
\text { qualité }\end{array}$ & $\begin{array}{c}\text { Temps } \\
\text { (heure)/ } \\
\text { jour }\end{array}$ & $\begin{array}{c}\text { Durée } \\
\text { d'amortissement } \\
\text { (année) }\end{array}$ & $\begin{array}{c}\text { Nombre } \\
\text { d'opérateurs }\end{array}$ \\
\hline \multicolumn{7}{|c|}{ Producteurs } \\
\hline Préparation du sol & Elevée & $\mathrm{Na}$ & Oui & 6 & 8 & 2 \\
\hline Labour & Elevée & Oui & Oui & 6 & 8 & 2 \\
\hline Semis/Repiquage & Moyenne & Oui & Oui & 5 & 8 & 2 \\
\hline Engrais/fumier & Moyenne & Oui & Oui & 5 & 7 & 1 \\
\hline Désherbage/Sarclage & Elevée & Oui & Oui & 5 & 8 & 2 \\
\hline Herbicidage & Moyenne & Oui & Oui & 5 & 7 & 1 \\
\hline $\begin{array}{l}\text { Lutte } \\
\text { insectes/maladies }\end{array}$ & Elevée & Oui & Oui & 5 & 7 & 1 \\
\hline Lutte ravageurs & Elevée & Oui & $\mathrm{Na}$ & 6 & 11 & 1 \\
\hline Récolte & Elevée & Oui & Oui & 6 & 8 & 2 \\
\hline Battage/Despathage & Elevée & Oui & Oui & 5 & 8 & 2 \\
\hline Nettoyage/vannage & Elevée & Oui & Oui & 5 & 8 & 2 \\
\hline Séchage & Elevée & Oui & Oui & 5 & 9 & 2 \\
\hline Décorticage/Egrenage & Elevée & Oui & Oui & 5 & 8 & 2 \\
\hline Conditionnement & Elevée & Oui & Oui & 5 & 8 & 2 \\
\hline \multicolumn{7}{|c|}{ Transformateurs } \\
\hline Epluchage & Elevée & Oui & Oui & 4 & 9 & 2 \\
\hline Nettoyage/Vannage & Elevée & Oui & Oui & 4 & 7 & 2 \\
\hline Lavage & Moyenne & $\mathrm{Na}$ & Oui & 3 & 8 & 2 \\
\hline Râpage & Elevée & Oui & Oui & 3 & 9 & 2 \\
\hline Pressage & Elevée & Oui & Oui & 4 & 9 & 2 \\
\hline Fermentation & Faible & $\mathrm{Na}$ & Oui & 4 & 8 & 2 \\
\hline Torréfaction & Elevée & Oui & Oui & 4 & 7 & 1 \\
\hline Séchage & Moyenne & $\mathrm{Na}$ & Oui & 5 & 8 & 2 \\
\hline Broyage & Elevée & Oui & Oui & 4 & 8 & 2 \\
\hline Décorticage/Egrenage & Elevée & Oui & Oui & 4 & 9 & 2 \\
\hline
\end{tabular}

Décorticage/Egrenage

Na : Non applicable

Légende :

Matériels et équipements performants et efficients
Matériels et équipements de capacité moyenne
Matériels et équipements à faible capacité




\section{DISCUSSION}

L'accroissement de la production agricole est l'une des préoccupations prioritaires des gouvernants afin de satisfaire la demande alimentaire de plus en plus croissante due à la démographie galopante. Cette satisfaction ne peut donc se faire sans, entre autres, l'augmentation des superficies agricoles, l'augmentation de la production, de la productivité (terres, main d'œuvre) et de la réduction des coûts de production (Havard, 2010). Par conséquent, l'atteinte des objectifs de production passe par l'utilisation des machines agricoles du fait de la pénibilité des opérations agricoles. Malgré les efforts consentis dans le sous-secteur de la mécanisation agricole, les producteurs et transformateurs sont partagés entre satisfaction et insatisfaction par rapport aux matériels et équipements utilisés. Ce qui suppose que les attentes des producteurs par rapport aux caractéristiques (capacité/puissance, temps de travail, durée de renouvellement etc.) de ces machines agricoles, sont faiblement ou pas prises en compte. Ces résultats rejoignent ceux de Hinnou (2012) pour qui, l'accent a été mis aux premières années de la mécanisation agricole, sur les approches directives (« Topdown ») sans tenir compte des besoins réels des agriculteurs. Les technologies ainsi issues de ces approches n'ont eu que peu d'effets sur les conditions de vie des agriculteurs (Hinnou, 2012) faute d'une adéquation entre les besoins et l'offre en technologie.

Les principales opérations culturales pré-récolte à mécaniser dans la zone d'étude selon les acteurs interviewés sont notamment la préparation du sol, le labour, l'herbicidage, le désherbage/Sarclage et la récolte. En effet, pour Agbangba et al. (2018), ces opérations constituent des étapes phares de la phase de pré-production et s'avèrent trop contraignantes pour la grande majorité des producteurs. De même, pour Havard (2010), l'utilisation des matériels et équipements facilite la réalisation des opérations exigeantes en énergie comme les travaux du sol, améliore les performances des opérations demandant de la technicité comme le semis et les sarclages, permet d'accroître les superficies cultivées et de valoriser le travail humain grâce à des tâches moins pénibles ou plus productives. En ce qui concerne les opérations post-récolte, la mécanisation du décorticage/Egrenage, du battage/despathage, de l'épluchage, du nettoyage/vannage, du pressage, du broyage, $\mathrm{du}$ conditionnement/emballage et du transport s'avère imminente. Ces résultats sont concordants avec ceux trouvés par Savi et Adégbola (2002) sur la caractérisation des unités de production. Pour ces derniers, en raison de la grande ambition dévéloppée par les unités de production et des aides considérables des institutions et organisations, des activités autrefois traditionnelles commencent par être mécanisées. Ainsi, les opérations mécanisées sont le râpage et le pressage. De même, pour Adegbola et al. (2006), l'utilisation du paquet technologique de semi-mécanisation de la production du gari a impacté positivement sur les conditions de vie des transformatrices mais aussi sur celles des producteurs de manioc. De plus, pour Ahouandjinou et al. (2010), la semimécanisation du procédé de production du beurre de karité a permis la réduction de la pénibilité de l'activité et l'accroissement du revenu des transformatrices. D'où la nécessité de mettre à la disposition des producteurs et transformateurs des matériels et équipements répondant à leurs attentes et besoins pour réduire la pénibilité des opérations culturales (Silou, 2003).

Par ailleurs, les producteurs et transformateurs ont une préférence pour des matériels et équipements dotés d'une grande autonomie journalière, une durée d'amortissement de huit ans et deux personnes au plus pour leur fonctionnement. De plus, ces équipements doivent présenter une puissance élevée, une capacité de réduction du taux de perte et d'amélioration de la qualité du produit obtenu. Pour Reboul (1960), le temps nécessaire pour labourer dépend de la parcelle (sol, topographie), de la puissance de la traction, de la vitesse d'avancement, de la largeur et de la profondeur de travail. De même, Adegbola et al. (2006) dans leur étude sur l'évaluation de l'impact socio-économique de la semi-mécanisation du procédé de production du gari au Bénin, ont montré que le 
paquet technologique de semi-mécanisation a permis d'améliorer de la qualité du produit final, d'accroître la production annuelle en gari et du revenu annuel aussi bien des transformateurs que des producteurs.

\section{Conclusion}

La mécanisation de l'agriculture constitue indéniablement de nos jours l'un des principaux enjeux pour la planification et le développement de la nation béninoise. Toutefois, il est important de prendre en compte les préférences exprimées par les producteurs et les transformateurs. A cet effet, l'analyse des besoins en matériels et équipements pré et post-récolte basée sur les logiques paysannes a permis de reconstituer les logiques socialement construites de la mécanisation agricole et d'évaluer le niveau de satisfaction des producteurs et transformateurs par rapport aux matériels et équipements. En revanche, les besoins en mécanisation restent grands et se traduisent par le nombre impressionnant des opérations agricoles qui nécessitent des actions. Autrement, les producteurs et les transformateurs préfèrent des équipements plus performants et moins contraignants. Somme toute, cette étude est indispensable à tout processus de mécanisation en ce sens qu'elle apporte une contribution à l'identification participative des opérations culturales et post culturales à mécaniser d'une part, mais aussi des besoins en termes d'équipements et matériels agricoles, d'autres part. La prise en considération de ces besoins dans le processus de développement et de diffusion des équipements agricoles aurait une répercution positive sur l'accroissement de la production et de la productivité agricoles. En conséquence, le pays pourrait d'une part, améliorer le niveau de sécurité alimentaire et de revenus des ménages. De plus, l'amélioration des conditions de vie et de travail, va inciter la jeune génération à s'adonner à l'agriculture. Il est donc du ressort des chercheurs et des décideurs de prendre en considération les besoins exprimés par les producteurs et transformateurs dans la mise au point des matériels et équipements agricoles conformément aux prototypes décrits.

\section{CONFLIT D'INTÉRÊTS}

Les auteurs déclarent qu'il n'y a aucun conflit d'intérêts.

\section{CONTRIBUTIONS DES AUTEURS}

CLH a participé à l'élaboration du protocole, l'analyse des données, la rédaction $\mathrm{du}$ projet de manuscrit et la correction du manuscrit ; RNAA a participé à la correction du manuscrit; VDA a participé à la collecte de données, l'analyse de données, la rédaction et la correction du manuscrit.

\section{REMERCIEMENTS}

Les auteurs tiennent à adresser leurs remerciements au Programme Cadre d'Appui à la Diversification Agricole (ProCAD) qui a financé la présente recherche. Ils remercient également tous les acteurs notamment les producteurs et les transformateurs pour leur disponibilité et les informations fournies.

\section{REFERENCES}

Adegbola Y, Maboudou A, Agli C, Adekambi A. 2006. Evaluation de l'impact socioéconomique de la semi-mécanisation du procédé de production du gari au Bénin. Institut National des Recherches Agricoles du Bénin/CRA-A/PAPA.

Adekunle A, AEHC, Oluwatosin A, Propcomm. 2015. Mécanisation agricole. Document de référence pour un plan d'action pour la transformation de l'agriculture africaine.

Agbangba C, Adegbola Y, Dagbenonbakin D, Houssou P, Ogouvide F, Agossou H. 2018. Analyse des besoins en équipements et matériels de production des spéculations des pôles de développement agricole du Bénin. INRAB/PAPAPE.

Ahouandjinou M, Adegbola Y, Yabi J, Adekambi A. 2010. Adoption et impact socio-économique de la semimécanisation du procédé de transformation des amandes de karité en beurre au nord-Bénin. Contributed Paper presented at the Joint 3rd African Association of Agricultural Economists (AAAE) and 48th Agricultural 
Economists Association of South Africa (AEASA) Conference. Cape Town. South Africa.

Atidegla C, Sintondji L, Hounkpe J, Kpadonou E. 2017. Effets du labour mécanisé successif sur le statut nutritif du sol et le rendement du Riz pluvial dans la commune d'Abomey Calavi (Sud Bénin). European Scientific Journal, 13(30): 341. DOI: 10.19044/esj.2017.v13n30p341.

Balse M, Ferrier C, Girard P, Hvard M, Herbel D. 2015. Une expérience originale de mécanisation partagée en Afrique. Les coopératives d'utilisation de matériel agricole du Bénin.

Boudon R. 2002. Théorie du choix rationnel ou individualisme méthodologique? Sociologie et Sociétés, 34(1) : 9-34.

FAO, Commission de la CEDEAO. 2018. Profil national genre des secteurs de l'agriculture et du développement ruralBénin. Serie des évaluations genre des pays. Cotonou.148PP.

Ficou M. 2017. La productivité agricole en Afrique stagne à cause du faible niveau de mécanisation.

Havard M. 2010. Etudes des besoins, des choix et de la gestion des équipements de motorisation agricole.

Hinnou C. 2012. Processus d'innovation endogène dans la riziculture au centre $\mathrm{du}$ Bénin. Mémoire DEA.

Hinnou C. 2018. Réseau social, gouvernance et innovations dans les chaînes de valeur du riz local au Bénin : Cas du département des Collines. Thèse de doctorat de l'Université d'Abomey-Calavi, 258p.

INSAE. 2016. Effectifs de la population des villages et quartiers de ville du Bénin (RGPH-4, 2013). Primature, République du Bénin, UNICEF, UNFPA. 85 p.

Paillé P, Mucchielli A. 2013. L'Analyse Qualitative en Sciences Humaines et Sociales. Armand Colin : Paris.

PNUD. 2015. Rapport National sur le Développement Humain 2015 : Agriculture, Sécurité alimentaire et Développement Humain au Bénin, 141p.

Reboul C. 1960. L'évaluation des besoins en travail sur une exploitation agricole. Centre National de la Comptabilité et d'Economie rurale.

Savi A, Adégbola Y. 2002. Caractérisation des unités de production du gari.

Side C. 2013. Stratégie de mécanisation de l'agriculture familiale en Afrique subsaharienne inclus étude de cas du Burkina Faso.

Silou T. 2003. Besoins et offre de technologie post-récolte dans l'agroalimentaire en Afrique subsaharienne: Rôle des technologues dans le développement de la petite entreprise. 2eme atelier international Voies alimentaires d'amélioration des situations nutritionnelles, Ouagadougou, 2328/11/2003. 